\title{
OPTIMAL INVESTMENT UNDER BEHAVIORAL CRITERIA IN INCOMPLETE DIFFUSION MARKET MODELS*
}

\author{
M. RÁSONYI ${ }^{\dagger}$ AND J. G. RODRÍGUEZ-VILLARREAL ${ }^{\ddagger}$
}

\begin{abstract}
The existence of optimal strategies is established for a behavioral investor in certain incomplete continuous time market models.

Key words. theory of cumulative perspectives, incomplete diffusion markets, independence, Krylov theorem

DOI. $10.1137 /$ S0040585X97T987892
\end{abstract}

1. Introduction. The most commonly accepted model for investors' preferences is expected utility theory, going back to [2], [19]. According to the tenets of this theory, an investor prefers a random return $X$ to $Y$ if $\mathbf{E} u(X) \geqq \mathbf{E} u(Y)$ for some utility function $u: \mathbf{R} \rightarrow \mathbf{R}$ that is usually assumed nonincreasing and concave. More recently, other theories have emerged and pose new challenges to mathematics.

The present paper treats preferences of cumulative prospect theory (CPT) [11], [18], where an "S-shaped" $u$ is considered (i.e., convex up to a certain point and concave from there on). Also, distorted probability measures are applied for calculating the utility of a given position with respect to a (possibly random) benchmark $G$. We remark that techniques of the present paper easily carry over to other types of preferences, too, such as rank-dependent utility [14] or acceptability indices [8].

The theory of optimal portfolio choice for CPT preferences is yet in its infancy. Continuous-time studies almost always assume a complete market model [3], [10], [7], [5], [16]. Only very specific types of incomplete continuous-time models have been treated to date (finite mixtures of complete models, the case where the price is a martingale under the physical measure, the case where the market price of risk is deterministic); see [17], [15]. In the present paper we take a step forward and consider incomplete models of a diffusion type where the return of the investment under consideration depends on some economic factors. Our main result asserts, under mild assumptions, the existence of an optimal strategy when the driving noise of the economic factors is independent of that of the investment and the rate of return is nonnegative. The independence condition is, admittedly, rather stringent and does not allow a leverage effect (see [4]). We are also able to accommodate models of a specific type where the factor may have nonzero correlation with the investment. We think that our results open the door to further generalizations.

2. Optimal investment model under behavioral criteria. In this section definitions and notation related to the problem of behavioral optimal investment are presented, based on [12], [6].

*Received by the editors December 23, 2014. This work was supported by the "Lendület" grant LP2015-6/2015 of the Hungarian Academy of Sciences, grants of CONACYT, Mexico, and of the University of Edinburgh. Part of this work was done while the first author was affiliated with the University of Edinburgh. Originally published in the Russian journal Teoriya Veroyatnostei $i$ ee Primeneniya, 60 (2015), No. 4, pp. 720-739.

http://www.siam.org/journals/tvp/60-4/T98789.html

$\dagger$ Alfréd Rényi Institute of Mathematics, Hungarian Academy of Sciences, Budapest, Hungary (rasonyi@renyi.mta.hu).

${ }^{\ddagger}$ University of Edinburgh, Edinburgh, UK. 
Unfortunately, most of the techniques developed in the literature for finding optimal policies rely on either the Markovian nature of the problem or on convex duality. These are no longer applicable under behavioral criteria. For this reason we shall consider a weak-type formulation of the control problem associated with optimal investment (subsection 2.1). Introducing a relaxation of the problem for which results in [12] apply, we can prove the existence of an optimal investment strategy (subsection 2.3).

2.1. The setting: Market and preferences. Fix a finite horizon $T>0$. We consider a financial market consisting of a risky asset, whose discounted price $\left(S_{t}\right)_{0 \leqq t \leqq T}$ depends on economic factors. These factors are described by a $d$-dimensional stochastic process $\left\{Y_{t}\right\}_{0 \leqq t \leqq T}$. Without entering into rigorous definitions at this point, our market model is described by the equations

$$
\begin{gathered}
d Y_{t}=\nu_{t}(Y .) d t+\kappa_{t}(Y .) d B_{t}, \quad Y_{0}=y, \\
d S_{t}=\theta_{t}(Y .) S_{t} d t+\lambda_{t}(Y .) S_{t} d W_{t}, \quad S_{0}=s>0,
\end{gathered}
$$

with $B, W$ independent standard Brownian motions of appropriate dimensions.

We also assume that there is a riskless asset of constant price equal to 1 . We shall be more specific later in this section. Stochastic volatility models provide prime examples of financial market models with dynamics (2.1) and (2.2); see [9].

The investor trades in the risky and riskless assets, investing a proportion $\phi_{t} \in$ $[0,1]$ of wealth into the risky asset at time $t$. This leads to the following equation for the wealth of the investor at time $t$ :

$$
d X_{t}=\phi_{t} \theta_{t}(Y .) X_{t} d t+\phi_{t} \lambda_{t}(Y .) X_{t} d W_{t}, \quad X_{0}=x,
$$

where $x>0$ is the investor's initial capital.

Borrowing and short selling are not allowed; hence, $\phi_{t}$ is a process taking values in $[0,1]$. We note that, in this model, the risky asset's price has no influence on the economic factors. We will see in section 3 how this assumption can be weakened.

We will need certain closedness results on the laws of Itô processes from [12]; hence, it is necessary to work in the "weak" setting of stochastic control theory, where the underlying probability space is not fixed.

We first set the requirements for the coefficients in (2.1), (2.3). Let $C\left([0, T] ; \mathbf{R}^{n}\right)$ denote the space of $\mathbf{R}^{n}$-valued continuous functions on $[0, T]$.

Denote by $p_{t}: C\left([0, T], \mathbf{R}^{d}\right) \rightarrow \mathbf{R}^{d}$ the projections $p_{t}(x)=.x_{t}$ and define the $\sigma$-algebras $\mathcal{N}_{t}=\sigma\left(\left\{p_{s}: s \leqq t\right\}\right)$ and $\mathcal{N}=\sigma\left(\left\{p_{s}: s \leqq T\right\}\right)$.

Definition 2.1. A process $\nu_{t}$ is $\mathbf{R}^{d}$-valued progressively measurable on the filtered space $\left(C\left([0, T] ; \mathbf{R}^{d}\right), \mathcal{N}_{T},\left\{\mathcal{N}_{t}\right\}_{0 \leqq t \leqq T}\right)$. We shall denote this functional by either $\nu_{t}(y$. or $\nu(t, y$.$) . Similarly, we define the coefficients \theta, \lambda, \kappa$ with the same measurability properties as $\nu$, but with values in $\mathbf{R}, \mathbf{R}$, and $S_{+}^{d}$, respectively, where $S_{+}^{d}$ denotes the set of real, symmetric, and positive semidefinite $d \times d$ matrices.

Definition 2.2. Let $x>0, y \in \mathbf{R}^{d}$ be fixed. An investment strategy $\pi$ is given by the following collection:

$$
\pi:=\left(\Omega, \mathcal{F},\left\{\mathcal{F}_{t}\right\}_{0 \leqq t \leqq T}, \mathbf{P}, X_{t}, Y_{t}, \phi_{t},\left(B_{t}, W_{t}\right)\right),
$$

where 
(a) $\left(\Omega, \mathcal{F},\left\{\mathcal{F}_{t}\right\}_{0 \leqq t \leq T}, \mathbf{P}\right)$ is a complete filtered probability space whose filtration satisfies the usual conditions;

(b) the process $\left(B_{t}, W_{t}\right)_{t \geqq 0}$ is a standard $(d+1)$-dimensional $\mathcal{F}_{t}$-Wiener process $\left(W_{t}\right.$ is unidimensional);

(c) $\phi: \Omega \times[0, T] \rightarrow[0,1]$ is $\mathcal{F} \otimes \mathcal{B}([0, T])$-measurable and $\mathcal{F}_{t}$-adapted;

(d) on the filtered probability space, $X_{t}, Y_{t}$ are $\mathcal{F} \otimes \mathcal{B}([0, T])$-measurable and $\mathcal{F}_{t^{-}}$ adapted processes such that for $t \in[0, T]$

$$
\begin{aligned}
& Y_{t}=y+\int_{0}^{t} \nu_{s}(Y .) d s+\int_{0}^{t} \kappa_{s}(Y .) d B_{s} \\
& X_{t}=x+\int_{0}^{t} \phi_{s} \theta_{s}(Y .) X_{s} d s+\int_{0}^{t} \phi_{s} \lambda_{s}(Y .) X_{s} d W_{s} .
\end{aligned}
$$

In other words, $\left(\Omega, \mathcal{F},\left\{\mathcal{F}_{t}\right\}_{0 \leqq t \leqq T}, \mathbf{P}, X_{t}, Y_{t},\left(B_{t}, W_{t}\right)\right)$ is a weak solution of the system of equations (2.1), (2.3). The process $\phi_{t}$ represents a ratio of investment in the risky asset, and it is measurable and $\mathcal{F}_{t}$-adapted. We do not consider the price process $S_{t}$ from (2.2) at all since it is enough to work with the "controlled dynamics" $X_{t}$.

When needed, we will use the notation $X^{\pi}, Y^{\pi}$, etc. to indicate that the object we mean belongs to $\pi$. Let $\Pi=\Pi(x, y)$ denote the collection of all strategies.

Assumption 2.1. The functional $\theta$ is nonnegative, i.e., $\theta(t, y) \geqq$.0 for all $t \in \mathbf{R}_{+}$ and $y \in C\left([0, T] ; \mathbf{R}^{d}\right)$.

Remark 2.1. In other words, the return of the risky asset must be nonnegative. This looks like a rather harmless assumption. On the other hand, as mentioned before, (b) in Definition 2.2 is stringent. It excludes the "leverage effect," namely, a commonly encountered phenomenon in the market in which the stock prices and its volatility are (negatively) correlated. Condition (b) can be relaxed; see section 3 .

We now present the framework of optimal investment under CPT, as proposed in [18]. We follow closely the notions developed in [15] and [6].

The investor assesses strategies by means of utilities on gains and losses, which are described in terms of functions $u_{ \pm}: \mathbf{R}_{+} \rightarrow \mathbf{R}_{+}$, by a reference point $G$ and functions $w_{ \pm}:[0,1] \rightarrow[0,1]$. The latter functions $w_{ \pm}$are introduced with the aim of explaining the distortions of investors' perception of the "likelihood" of gains and losses.

According to the tenets of CPT, investors use benchmarks to assess the portfolio outcomes; this is modeled by a real-valued random variable $G$. The quantity $G$ depends on economic factors as follows: let us denote by $F$ a fixed deterministic functional $F: C\left([0, T] ; \mathbf{R}^{d}\right) \rightarrow \mathbf{R}_{+}$which is $\mathcal{N}_{T}$-measurable. As the probability space is not fixed, for each $\pi \in \Pi$ we define the corresponding reference point by $G^{\pi}:=$ $F\left(Y^{\pi}\right)$. That is, we assume that the benchmark is a nonnegative functional of the economic factors. Results can easily be extended to the slightly more general case where $G^{\pi}:=F\left(Y^{\pi}, B^{\pi}\right)$ for some functional $F$.

Now we describe the optimization problem with which we are mainly concerned. As in expected utility theory, we aim to maximize a function depending on an investor's wealth and on the elements described above: $u_{ \pm}, w_{ \pm}$, and the investor's reference point $G$. For any strategy $\pi \in \Pi$, we define the functionals

$$
\begin{aligned}
& V_{+}(\pi):=\int_{0}^{\infty} w_{+}\left(\mathbf{P}^{\pi}\left(u_{+}\left(\left(X_{T}^{\pi}-G^{\pi}\right)_{+}\right)>t\right)\right) d t, \\
& V_{-}(\pi):=\int_{0}^{\infty} w_{-}\left(\mathbf{P}^{\pi}\left(u_{-}\left(\left(X_{T}^{\pi}-G^{\pi}\right)_{-}\right)>t\right)\right) d t .
\end{aligned}
$$

Copyright (C) by SIAM. Unauthorized reproduction of this article is prohibited. 
The optimal portfolio problem for an investor under CPT consists of maximizing the following performance functional:

$$
V(\pi):=V_{+}(\pi)-V_{-}(\pi)
$$

which is defined provided that at least one of the summands is finite. Set $\Pi^{\prime}:=\{\pi \in$ $\left.\Pi: V_{-}(\pi)<\infty\right\}$ and define

$$
V:=\sup _{\pi \in \Pi^{\prime}} V(\pi)
$$

The value $V$ represents the maximal satisfaction achievable by investing in the stock and riskless asset in a CPT framework. Our purpose is to prove, under some assumptions, the existence of $\widehat{\pi} \in \Pi^{\prime}$ such that $V(\widehat{\pi})=V$.

2.2. Main result. We make the following assumptions. Recall the notation $y_{t}^{\star}=\sup _{s \leqq t}\left|y_{s}\right|$.

Assumption 2.2. The functionals $\lambda:[0, T] \times C\left([0, T] ; \mathbf{R}^{d}\right) \rightarrow \mathbf{R}$ and $\theta:[0, T] \times$ $C\left([0, T] ; \mathbf{R}^{d}\right) \rightarrow \mathbf{R}$ are uniformly bounded.

The functionals $\kappa$ and $\nu$ satisfy a linear growth condition; i.e., there is a constant $K>0$ such that for any $t \in[0, T]$ and $y \in C\left([0, T] ; \mathbf{R}^{d}\right)$,

$$
\left\|\kappa_{t}(y .)\right\| \leqq K\left(1+\left|y_{t}\right|\right) \quad \text { and } \quad\left|\nu_{t}(y .)\right| \leqq K\left(1+\left|y_{t}\right|\right) .
$$

Furthermore, for fixed $t \geqslant 0$ and functions $y^{n}, z \in C\left([0, T] ; \mathbf{R}^{d}\right)$ such that $\left(y^{n}-z\right)_{t}^{\star} \rightarrow$ $0, n \rightarrow \infty$ we have $\kappa_{t}\left(y^{n}\right) \rightarrow \kappa_{t}(z$.$) , and the same holds for the functionals \lambda, \theta$, and $\nu$. We will refer to this as the coefficients being path-continuous at any time $t \in[0, T]$.

Assumption 2.3. A (weak) solution of (2.4) exists and is unique in law.

Assumption 2.4. The functions $u_{ \pm}: \mathbf{R}_{+} \rightarrow \mathbf{R}_{+}$and $w_{ \pm}:[0,1] \rightarrow[0,1]$ are continuous and nondecreasing with $u_{ \pm}(0)=0, w_{ \pm}(0)=0, w_{ \pm}(1)=1$, and

$$
\begin{aligned}
u_{+}(x) \leqq k_{+}\left(x^{\alpha}+1\right) & \text { for all } x \in \mathbf{R}_{+}, \\
w_{+}(p) \leqq g_{+} p^{\gamma} & \text { for all } p \in[0,1]
\end{aligned}
$$

with $\gamma, \alpha, k_{+}, g_{+}>0$ fixed constants.

We denote by $L^{p}(\Omega, \mathbf{P})$ the usual space of $p$-integrable random variables on a probability space $(\Omega, \mathcal{F}, \mathbf{P})$.

Assumption 2.5. There is $\psi>0$ such that $G^{\pi} \in L^{\psi}\left(\Omega, \mathbf{P}^{\pi}\right)$ for all $\pi \in \Pi$.

Note that under Assumption 2.3, the law of $G^{\pi}$ is independent of $\pi$, and hence Assumption 2.5 holds if and only if $G^{\pi} \in L^{\psi}\left(\Omega, \mathbf{P}^{\pi}\right)$ for one particular $\pi$. We set $\vartheta:=\psi / \gamma$.

In order to ensure that the functional $V$ and the optimization problem in (2.9) are defined over a nonempty set, we introduce the following assumption on $u_{-}$, the distortion function $w_{-}$, and the reference point $G^{\pi}$.

Assumption 2.6. The functions $w_{-}, u_{-}$are such that for all $\pi \in \Pi$,

$$
\int_{0}^{\infty} w_{-}\left(\mathbf{P}^{\pi}\left(u_{-}\left(G^{\pi}\right)>y\right)\right) d y<\infty
$$

This assumption ensures that the set $\Pi^{\prime}$ is not empty. Let $\left(\Omega, \mathcal{F},\left\{\mathcal{F}_{t}\right\}_{0 \leqq t \leqq T}, \mathbf{P}\right)$ be a filtered probability space, where $(2.1)$ has a solution $Y_{t}$. Then setting $\phi_{t}:=0$ and $X_{t}:=x$ for all $t,\left(\Omega, \mathcal{F},\left\{\mathcal{F}_{t}\right\}_{0 \leqq t \leqq T}, \mathbf{P}, x, Y_{t}, 0,\left(B_{t}, W_{t}\right)\right)$ belongs to $\Pi^{\prime}$. 
Remark 2.2. As $u_{ \pm}$and $w_{ \pm}$are nondecreasing, under Assumption $2.6 \Pi^{\prime}=\Pi$ by Remark 2.6 below.

Our main result can now be stated.

Theorem 2.1. Under Assumptions 2.1-2.3 the problem (2.9) is well-posed, i.e., $V<\infty$. Moreover, there exists an optimal strategy $\widehat{\pi} \in \Pi^{\prime}$ attaining the supremum in (2.9), i.e., $V=V(\widehat{\pi})$.

2.3. A relaxation of the set of controls. We introduce a relaxation of the problem by extending the class of investment strategies given in Definition 2.2; we shall call this extension the class of auxiliary controls. This relaxation is introduced in order to ensure the closedness of the set of laws of the processes $\left\{\left(Y^{\pi}, X^{\pi}{ }^{\pi}\right): \pi \in \bar{\Pi}\right\}$ and $\Pi \subset \bar{\Pi}$.

The coefficients of the process $\left(Y_{t}, X_{t}\right)$ appear in the martingale problem formulation of (2.4) and (2.5) as coefficients of a differential operator (see Definition 2.4 in what follows). In order to use results on compactness of laws [12], we shall consider a "convex extension" of the sets in which the coefficients in (2.4) and (2.5) take values.

Definition 2.3. Denote by $\mathbf{A}$ the set $S_{+}^{d+1} \times \mathbf{R}^{d+1}$. For any pair of continuous functions $(x, y.) \in C\left([0, T] ; \mathbf{R}_{+} \times \mathbf{R}^{d}\right)$ and for any $t \in[0, T]$ we define

$$
\begin{array}{r}
A_{t}(x, y .)=\left\{(a, b) \in \mathbf{A} \mid a=\left(\begin{array}{cc}
\kappa \kappa^{\star}(t, y .) / 2 & 0 \\
0 & m \lambda^{2}(t, y .) x_{t}^{2} / 2
\end{array}\right),\right. \\
\left.b=\left(\begin{array}{c}
\nu(t, y .) \\
l \theta(t, y \cdot) x_{t}
\end{array}\right), \quad 0 \leqq m \leqq 1,0 \leqq l \leqq \sqrt{m}\right\} .
\end{array}
$$

Remark 2.3. Notice that for any investment strategy $\pi$ as in Definition 2.2, if

$$
\sigma_{t}=\left(\begin{array}{cc}
\kappa(t, y .) & 0 \\
0 & \phi_{t} \lambda(t, y .) x_{t}
\end{array}\right), \quad b_{t}=\left(\begin{array}{c}
\nu(t, y .) \\
\phi_{t} \theta(t, y \cdot) x_{t}
\end{array}\right)
$$

then, defining $a_{t}=\frac{1}{2} \sigma_{t} \sigma_{t}^{\star}$, the pair $\left(a_{t}, b_{t}\right)$ belongs to $A_{t}(x, y$. $)$.

The following definition describes the family of auxiliary controls used throughout this work. It stresses the fact of having Itô processes whose coefficients belong to the convex sets $A_{t}(x, y$.$) in "a measurable way" as t, x$., and $y$. vary.

DeFinition 2.4. We define a family of auxiliary controls $\bar{\Pi}=\bar{\Pi}(x, y)$. Namely, an auxiliary control $\bar{\pi} \in \bar{\Pi}$ consists of a collection

$$
\bar{\pi}:=\left(\Omega, \mathcal{F},\left\{\mathcal{F}_{t}\right\}_{0 \leqq t \leqq T}, \mathbf{P}, X_{t}, Y_{t},\left(B_{t}, W_{t}\right)\right),
$$

where

(a) $\left(\Omega, \mathcal{F},\left\{\mathcal{F}_{t}\right\}_{0 \leqq t \leq T}, \mathbf{P}\right)$ is a complete filtered probability space whose filtration satisfies the usual conditions;

(b) the process $\xi_{t}:=\left(B_{t}, W_{t}\right)$ is an $\mathbf{R}^{d+1}$-valued standard $\mathcal{F}_{t}$-Wiener process $\left(W_{t}\right.$ is unidimensional);

(c) there exists an $\mathbf{A}$-valued, $\mathcal{F} \otimes \mathcal{B}([0, T])$-measurable and $\mathcal{F}_{t}$-adapted process, denoted by $\left(a_{t}, b_{t}\right)$, such that the following conditions hold:

(c1) the processes $X_{t}$ and $Y_{t}$ are $\mathcal{F} \otimes \mathcal{B}([0, T])$-measurable and $\mathcal{F}_{t}$-adapted such that a.s. for all $t \in[0, T]$,

$$
\left(\begin{array}{c}
Y_{t} \\
X_{t}
\end{array}\right)=\left(\begin{array}{c}
y \\
x
\end{array}\right)+\int_{0}^{t} \sqrt{2 a_{s}} d \xi_{s}+\int_{0}^{t} b_{s} d s
$$

(c2) for almost all $(\omega, t) \in \Omega \times[0, T]$, we have $\left(a_{t}, b_{t}\right) \in A_{t}(X ., Y$.). 
We will write $X^{\bar{\pi}}, Y^{\bar{\pi}}$ to indicate that $X, Y$ belong to $\bar{\pi}$.

For each $\bar{\pi} \in \bar{\Pi}$, we can define $V_{ \pm}(\bar{\pi})$ as before, and we can set $V(\bar{\pi}):=V_{+}(\bar{\pi})-$ $V_{-}(\bar{\pi})$ for $\bar{\pi} \in \bar{\Pi}^{\prime}:=\left\{\pi \in \bar{\Pi}: V_{-}(\pi)<\infty\right\}$.

Remark 2.4. For a pair of processes $a_{t}$ and $b_{t}$ in $A_{t}(X ., Y$.) one can define the corresponding real-valued processes $l_{t}$ and $m_{t}$ with $0 \leqq m_{t} \leqq 1,0 \leqq l_{t} \leqq \sqrt{m_{t}}$, setting

$$
l_{t}:=\frac{b_{t}^{d+1} 1_{\theta_{t}\left(t, Y_{t}\right) \neq 0}}{X_{t} \theta_{t}\left(t, Y_{t}\right)}, \quad m_{t}:=\frac{a^{d+1, d+1} 1_{\lambda_{t}\left(t, Y_{t}\right) \neq 0}}{X_{t}^{2} \lambda^{2}\left(t, Y_{t}\right)} .
$$

Conditions (c1) and (c2) in Definition 2.4, together with Assumption 2.2, imply that $l_{t}, m_{t}$ can be chosen $\mathcal{F} \otimes \mathcal{B}([0, T])$ measurable and $\mathcal{F}_{t}$-adapted.

Equation (2.14) can be rewritten as the set of equations in what follows. Denote

$$
\sigma_{t}=\left(\begin{array}{cc}
\kappa(t, Y .) & 0 \\
0 & \sqrt{m_{t}} \lambda(t, Y .) X_{t}
\end{array}\right), \quad b_{t}=\left(\begin{array}{c}
\nu(t, Y .) \\
l_{t} \theta(t, Y .) X_{t}
\end{array}\right) .
$$

We get

$$
\begin{aligned}
& Y_{t}=y+\int_{0}^{t} \nu_{s}(Y .) d s+\int_{0}^{t} \kappa_{s}(Y .) d B_{s} \\
& X_{t}=x+\int_{0}^{t} l_{s} \theta_{s}(Y .) X_{s} d s+\int_{0}^{t} \sqrt{m_{s}} \lambda_{s}(Y .) X_{s} d W_{s} .
\end{aligned}
$$

Definition 2.5. Let $\bar{\pi} \in \bar{\Pi}$ be an auxiliary control. We say that $X_{t}^{\bar{\pi}}$ is a portfolio value process if $l_{t}=\sqrt{m_{t}}$, i.e.,

$$
d X_{t}=\sqrt{m_{t}} \theta(t, Y .) X_{t} d t+\sqrt{m_{t}} \lambda(t, Y .) X_{t} d W_{t} .
$$

Remark 2.5. If $X_{t}^{\bar{\pi}}$ is a portfolio value process, then, taking $\phi_{t}=\sqrt{m_{t}}$, we can see that

$$
\left(\Omega^{\bar{\pi}}, \mathcal{F}^{\bar{\pi}},\left\{\mathcal{F}_{t}^{\bar{\pi}}\right\}_{t \geqq 0}, \mathbf{P}^{\bar{\pi}}, X_{t}^{\bar{\pi}}, Y_{t}^{\bar{\pi}}, \phi_{t},\left(B_{t}^{\bar{\pi}}, W_{t}^{\bar{\pi}}\right)\right)
$$

belongs to $\Pi$.

Remark 2.6. Suppose that we are given a $\bar{\pi} \in \bar{\Pi}$; i.e., there is a standard $(d+1)$-dimensional Brownian motion $(B, W)$ on $\left(\Omega^{\bar{\pi}}, \mathcal{F}^{\bar{\pi}},\left\{\mathcal{F}_{t}^{\bar{\pi}}\right\}_{t \geqq 0}, \mathbf{P}^{\bar{\pi}}\right)$ and processes $X_{t}^{\bar{\pi}}, Y_{t}^{\bar{\pi}}, m_{t}^{\bar{\pi}}, l_{t}^{\bar{\pi}}$ such that (2.15) and (2.16) hold. Define the continuous semimartingale

$$
M_{t}^{\bar{\pi}}:=\int_{0}^{t} \sqrt{m_{s}^{\bar{\pi}_{t}}} \lambda_{s}(Y .) d W_{s}+\int_{0}^{t} l_{s}^{\bar{\pi}_{t}} \theta_{s}(Y .) d s .
$$

Then we can rewrite $(2.16)$ as

$$
X_{t}=x+\int_{0}^{t} X_{s} d M_{s}^{\bar{\pi}}
$$

Equation (2.18) has a unique strong solution on the given probability space, namely, the stochastic exponential

$$
X_{t}^{\bar{\pi}}=x \exp \left\{\int_{0}^{t} \sqrt{m_{s}} \lambda_{s}\left(Y_{\cdot}^{\bar{\pi}}\right) d W_{s}^{\bar{\pi}}+\int_{0}^{t}\left[l_{s} \theta_{s}\left(Y_{\cdot}^{\bar{\pi}}\right)-\frac{1}{2} m_{s} \lambda_{s}^{2}\left(Y_{\cdot}^{\bar{\pi}}\right)\right] d s\right\},
$$

and this process is positive $\mathbf{P}^{\bar{\pi}}$-a.s.

Copyright $@$ by SIAM. Unauthorized reproduction of this article is prohibited. 


\subsection{Krylov's theorem and related results.}

Lemma 2.1. Let $M=\max \left\{\|\lambda\|_{\infty},\|\theta\|_{\infty}\right\}$, and let $K>0$ be such that

$$
\left|\nu_{t}(y .)\right| \vee\left\|\kappa_{t}(y .)\right\| \leqq K\left(1+\left|y_{t}\right|\right) \text {. }
$$

The set $A_{t}(x ., y$.$) is convex, closed, and bounded, where the bound depends on M, K$, $x_{t}$, and $y_{t}$ only.

Proof. Notation $|\cdot|$ will refer to Euclidean norms of varying dimensions. For simplicity, we assume $d=1$ and denote $\zeta_{t}=\left(x_{t}, y_{t}\right)$. Notice that

$$
\left|\left(\sigma_{t}, b_{t}\right)\right|=\left(\kappa_{t}^{2}(y .)+m^{2} \lambda_{t}^{2}(y .) x_{t}^{2}+\nu_{t}^{2}(y .)+l^{2} \theta_{t}^{2}(y .) x_{t}^{2}\right)^{1 / 2}
$$

hence

$$
\left|\left(\sigma_{t}, b_{t}\right)\right| \leqq K\left(1+\left|y_{t}\right|\right)+M\left|x_{t}\right|+K\left(1+\left|y_{t}\right|\right)+M\left|x_{t}\right| \leqq N\left(1+\left|x_{t}\right|+\left|y_{t}\right|\right)
$$

with $N=2 K \vee 2 M$. Then

$$
\left|\left(\sigma_{t}, b_{t}\right)\right| \leqq N^{\prime}\left(1+\left|\zeta_{t}\right|\right) .
$$

It is clear that the set is closed. For fixed $t, x$., and $y$. the set is bounded. Indeed, let $\left(a_{t}, b_{t}\right) \in A_{t}(x ., y$. $)$. Then we have

$$
\begin{aligned}
\left|\left(a_{t}, b_{t}\right)\right| & =\left(\frac{1}{4}\left(\kappa_{t}^{2}(y .)\right)^{2}+\frac{1}{4}\left(m^{2} \lambda_{t}^{2}(y .) x_{t}^{2}\right)^{2}+\nu_{t}^{2}(y .)+l^{2} \theta_{t}^{2}(y .) x_{t}^{2}\right)^{1 / 2}, \\
\left|\left(a_{t}, b_{t}\right)\right| & \leqq \frac{1}{2} K^{2}\left(1+\left|y_{t}\right|\right)^{2}+\frac{1}{2} M^{2}\left|x_{t}\right|^{2}+K\left(1+\left|y_{t}\right|\right)+M\left|x_{t}\right| \\
& \leqq K_{1}\left(1+\left|y_{t}\right|\right)^{2}+M_{1}\left(1+\left|x_{t}\right|\right)^{2}
\end{aligned}
$$

with $K_{1}=\frac{1}{2} K^{2}+K$ and $M_{1}=\frac{1}{2} M^{2} \vee 1$, so

$$
\left|\left(a_{t}, b_{t}\right)\right| \leqq \mathcal{N}\left(1+\left|\zeta_{t}\right|^{2}\right),
$$

with $\mathcal{N}$ depending only on $K$ and $M$. The set $A_{t}(x ., y$.) is also convex.

Let $(\alpha, b),(\gamma, c) \in A_{t}(x ., y$. $)$; then, for $0 \leq \mu \leq 1$,

$$
\begin{aligned}
& \mu(\alpha, b)+(1-\mu)(\gamma, c) \\
& =\left(\left(\begin{array}{cc}
\frac{1}{2} \kappa_{t}^{2}(y .) & 0 \\
0 & \frac{1}{2}(\mu m+(1-\mu) n) \lambda_{t}^{2}(y .) x_{t}^{2}
\end{array}\right),\left(\begin{array}{c}
\nu_{t}(y .) \\
(\mu l+(1-\mu) p) \theta_{t}(y .) x_{t}
\end{array}\right)\right),
\end{aligned}
$$

with $0 \leqq m, n \leqq 1,0 \leqq l \leqq \sqrt{m}$, and $0 \leqq p \leqq \sqrt{n}$. Clearly,

$$
\mu l+(1-\mu) p \leqq \sqrt{\mu m+(1-\mu) n}
$$

by concavity of the square root function. Lemma 2.1 is proved.

Remark 2.7. Introducing a new notation, we may summarize Lemma 2.1 as

$$
\left\|A_{t}(x ., y .)\right\|:=\max \left\{\left|\left(a_{t}, b_{t}\right)\right|:\left(a_{t}, b_{t}\right) \in A_{t}(x ., y .)\right\} \leqq K^{\prime}\left(1+\left|x_{t}\right|^{2}\right)
$$

for some $K^{\prime} \geq 0$.

Copyright $@$ by SIAM. Unauthorized reproduction of this article is prohibited. 
In order to deal with (semi)continuity issues related to the family of sets defined in Definition 2.3 and (2.13), the support functions of sets $A_{t}(x, y$.$) are now considered.$ We denote for all $u \in \mathbf{R}^{(d+1)(d+1)}, v \in \mathbf{R}^{d+1}$, and $t \in[0, T]$,

$$
F_{t}(x ., y .)(u, v)=\max \left\{\sum_{i, j} a_{i j} u_{i j}+\sum_{j} b_{j} v_{j}:(a, b) \in A_{t}(x ., y .)\right\} .
$$

Under Assumption 2.2, for fixed $t \geqq 0$ and $(u, v)$, the support function $(x, y.) \mapsto$ $F_{t}(x ., y).(u, v)$ is continuous, since we are fixing $t$, restricting the trajectories to $[0, t]$, and thus the max is taken over a compact set by Lemma 2.1. In particular, the set $A_{t}(x ., y$.$) is upper-semicontinuous in the sense of Assumption 3.1(iii) in [12].$

For fixed $u, v \in \mathbf{A},(t, x ., y.) \rightarrow F_{t}(x, y).(u, v)$ is a Borel function on $[0, T] \times$ $C\left([0, T] ; \mathbf{R}^{d+1}\right)$.

We now present some moment estimates which will, in particular, guarantee tightness of the family of laws of $\left(X^{\bar{\pi}}, Y^{\bar{\pi}}\right), \bar{\pi} \in \bar{\Pi}$ in $C\left([0, T] ; \mathbf{R}^{d+1}\right)$.

Proposition 2.1. For ease of reference we denote $\zeta_{t}^{\bar{\pi}}=\left(Y_{t}^{\bar{\pi}}, X_{t}^{\bar{\pi}}\right)$. Under Assumptions 2.2 and 2.3 , for any $m>0$,

$$
\sup _{\bar{\pi} \in \bar{\Pi}} \mathbf{E}_{\bar{\pi}}\left[\sup _{t \leqq T}\left|\zeta_{t}^{\bar{\pi}}\right|^{m}\right]<\infty .
$$

Proposition 2.2. Under Assumptions 2.2 and 2.3 , let $\bar{\pi} \in \bar{\Pi}$ and $\left(Y_{t}^{\bar{\pi}}, X_{t}^{\bar{\pi}}\right)$ its associated processes solve (2.15) and (2.16). Then there exists a constant $K>0$ not depending on $\bar{\pi} \in \bar{\Pi}$, such that for any $\eta>0$ and $s, t \in[0, T]$,

$$
\mathbf{E}_{\bar{\pi}}\left\|\zeta_{t}^{\bar{\pi}}-\zeta_{s}^{\bar{\pi}}\right\|^{\eta} \leqq K|t-s|^{\eta / 2}
$$

See the appendix for a standard proof of both propositions above. A well-known result on tightness of measures on $C\left([0, T] ; \mathbf{R}^{d+1}\right)$ gives the following corollary. This could also be obtained by the method of Theorem 3.2 in [13].

Corollary 2.1. Let Assumption 2.2 be in force. Let $\left\{\bar{\pi}_{n}\right\}_{n \in \mathbf{N}} \subset \bar{\Pi}$. The sequence of laws of the process $\zeta^{\bar{\pi}_{n}}$ on $C\left([0, T] ; \mathbf{R}^{d+1}\right)$ is relatively weakly compact.

Now we restate Theorem 3.2 of [12] in our setting, which will provide weak compactness of the distributions of weak controls.

Theorem 2.2. Let Assumption 2.2 be in force. Denote by $\mathbf{Q}^{\bar{\pi}}$ the distribution of $\zeta^{\bar{\pi}}$ on $C\left([0, T] ; \mathbf{R}^{d+1}\right)$. Then the set $\left\{\mathbf{Q}^{\bar{\pi}}: \bar{\pi} \in \bar{\Pi}\right\}$ is sequentially weakly compact: for any sequence $\bar{\pi}_{n} \in \bar{\Pi}$ there is a subsequence $n(m) \rightarrow \infty$ as $m \rightarrow \infty$ and $a \bar{\pi} \in \bar{\Pi}$ such that for any real-valued, bounded, continuous function $H(x$.$) on C\left([0, T] ; \mathbf{R}^{d+1}\right)$ we have

$$
\lim _{m \rightarrow \infty} \mathbf{E}_{\nu_{m}} H\left(\zeta^{\nu_{m}}\right)=\mathbf{E}_{\pi} H\left(\zeta^{\pi}\right)
$$

where $\nu_{m}=\pi_{n(m)}$.

Proof. It follows from the above discussions that Assumption 3.1(ii) and (iii) in [12] hold in the present case. One does not have Assumption 3.1(i) of [12], though (linear growth condition on $\left.\left\|A_{t}(X ., Y).\right\|\right)$; there is a quadratic growth instead (see (2.21)). But, as Corollary 2.1 shows, this is still sufficient to get tightness (and hence relative weak compactness) of the sequence $\mathbf{Q}^{\bar{\pi}_{n}}$ in our setting. Then one can check that the proof of Theorem 3.2 in [12] goes through, and we can conclude.

The following lemma shows that, for any auxiliary control $\bar{\pi}$ in the sense of Definition 2.4, we can associate an investment strategy (in the sense of Definition 2.2) with a higher value function. 
Lemma 2.2. Let

$$
\bar{\pi}=\left(\Omega^{\bar{\pi}}, \mathcal{F}^{\bar{\pi}},\left\{\mathcal{F}_{t}^{\bar{\pi}}\right\}_{0 \leqq t \leqq T}, \mathbf{P}^{\bar{\pi}},\left(X^{\bar{\pi}}, Y^{\bar{\pi}}\right),\left(B^{\bar{\pi}}, W^{\bar{\pi}}\right)\right) \in \bar{\Pi} .
$$

Then a solution to

$$
\begin{gathered}
d Y_{t}=\nu_{t}(Y .) d t+\kappa_{t}(Y .) d B_{t}, \quad Y_{0}=y \\
d \widehat{X}_{t}=\sqrt{m_{t}} \theta_{t}(Y .) \widehat{X}_{t} d t+\sqrt{m_{t}} \lambda_{t}(Y .) \widehat{X}_{t} d W_{t}, \quad X_{0}=x,
\end{gathered}
$$

exists on the same filtered probability space and $\widehat{X}_{T} \geqq X_{T}^{\bar{\pi}}$ a.s. Moreover, $\left\{\widehat{X}_{t}\right\}_{0 \leqq t \leqq T}$ is a portfolio value process.

Proof. Let us define

$$
Z_{t}:=\exp \left(-\int_{0}^{t}\left(l_{s}^{\bar{\pi}}-\sqrt{m_{s}^{\bar{\pi}}}\right)\left(X_{.}^{\bar{\pi}}, Y_{.}^{\bar{\pi}}\right) \theta\left(Y_{.}^{\bar{\pi}}\right) d s\right)
$$

and set $\widehat{X}_{t}:=Z_{t} X_{t}^{\bar{\pi}}$. Itô's formula shows that $\widehat{X}_{t}$ indeed verifies (2.27). Since $\theta_{t} \geqq 0$ was assumed, we get that $Z_{t} \geqq 1$, and hence $\widehat{X}_{t} \geqq X_{t}^{\bar{\pi}}$, for all $t$. Lemma 2.2 is proved.

2.5. Proof of Theorem 2.1. Let $t>0$. By (2.11) and (2.10),

$$
w_{+}\left(\mathbf{Q}^{\pi}\left(u_{+}\left(\left(X_{T}^{\pi}-G^{\pi}\right)_{+}\right)>t\right)\right) \leqq g_{+}\left[\mathbf{Q}^{\pi}\left(\left(X_{T}^{\pi}-G^{\pi}\right)_{+}^{\alpha}>\frac{t}{k_{+}}-1\right)\right]^{\gamma} .
$$

Hence,

$$
\begin{aligned}
& V_{+}(\pi) \leqq g_{+} \int_{0}^{\infty}\left[\mathbf{Q}^{\pi}\left(\left(X_{T}^{\pi}-G^{\pi}\right)_{+}^{\alpha}>\frac{t}{k_{+}}-1\right)\right]^{\gamma} d y \\
& =g_{+}\left(1+\int_{k_{+}}^{\infty}\left[\mathbf{Q}^{\pi}\left(\left(X_{T}^{\pi}-G^{\pi}\right)_{+}^{\alpha}>\frac{t}{k_{+}}-1\right)\right]^{\gamma} d y\right), \\
& \int_{k_{+}}^{\infty}\left[\mathbf{Q}^{\pi}\left(\left(X_{T}^{\pi}-G^{\pi}\right)_{+}^{\alpha}>\frac{t}{k_{+}}-1\right)\right]^{\gamma} d y \\
& \leqq k_{+} \int_{0}^{\infty}\left[\mathbf{Q}^{\pi}\left(\left(X_{T}^{\pi}-G^{\pi}\right)_{+}^{\alpha}>s\right)\right]^{\gamma} d s .
\end{aligned}
$$

If $s \geq 1$, applying Chebyshev's inequality and Assumption 2.5,

$$
\begin{aligned}
{\left[\mathbf{Q}^{\pi}\left(\left(X_{T}^{\pi}-G^{\pi}\right)_{+}^{\alpha}>s\right)\right]^{\gamma} } & =\left[\mathbf{Q}^{\pi}\left(\left(X_{T}^{\pi}-G^{\pi}\right)_{+}^{\alpha \vartheta}>s^{\vartheta}\right)\right]^{\gamma} \\
& \leqq \frac{\left[\mathbf{E}_{\pi}\left(X_{T}^{\pi}-G^{\pi}\right)_{+}^{\alpha \vartheta}\right]^{\gamma}}{s^{\vartheta \gamma}} \leqq M^{\gamma} \frac{1}{s^{\vartheta \gamma}},
\end{aligned}
$$

where $M=\sup _{\pi} \mathbf{E}_{\pi}\left(X_{T}^{\pi}\right)_{+}^{\alpha \vartheta}<\infty$ (note that $G \geqq 0$ ), by Proposition 2.1. Note that $1 / s^{\vartheta \gamma}$ is integrable on $[1, \infty)$.

Hence the problem is well-posed since $V(\pi) \leqq V_{+}(\pi)$ for all $\pi \in \Pi^{\prime}$, and we have just seen that the latter has an upper bound independent of $\pi$.

Copyright $@$ ㅇ by SIAM. Unauthorized reproduction of this article is prohibited. 
By Theorem 2.2, the set of laws $\left\{\mathbf{Q}^{\pi}\right\}, \pi \in \bar{\Pi}$, of the processes $\zeta^{\pi}=\left(X^{\pi}, Y^{\pi}\right)$ is relatively compact in the weak topology. Let $\left\{\pi^{n}\right\}$ be a sequence of controls $\pi^{n} \in \bar{\Pi}^{\prime}$ such that

$$
V\left(\pi^{n}\right) \rightarrow \sup _{\pi \in \bar{\Pi}^{\prime}} V(\pi), \quad n \rightarrow \infty
$$

There is a subsequence of $\left\{\pi^{n}\right\}$ denoted by $\left\{\pi^{k}\right\}$ such that $\mathbf{Q}^{\pi^{k}} \Rightarrow \mathbf{Q}^{\pi^{\star}}$ as $k \rightarrow \infty$ and $\pi^{\star} \in \bar{\Pi}$.

By Skorokhod's theorem there is a probability space $(\widetilde{\Omega}, \widetilde{\mathcal{F}}, \widetilde{\mathbf{P}})$ and random variables $\widetilde{X}^{k}, \widetilde{Y}^{k}:(\widetilde{\Omega}, \widetilde{\mathcal{F}}, \widetilde{\mathbf{P}}) \rightarrow C([0, T] ; \mathbf{R}), C\left([0, T] ; \mathbf{R}^{d}\right)$, respectively, such that the law of $\left(\widetilde{X}^{k}, \widetilde{Y}^{k}\right)$ equals $\mathbf{Q}^{\pi_{k}}$ and $\widetilde{X}, \widetilde{Y}:(\widetilde{\Omega}, \widetilde{\mathcal{F}}, \widetilde{\mathbf{P}}) \rightarrow C([0, T] ; \mathbf{R}), C\left([0, T] ; \mathbf{R}^{d}\right)$ with law equal to $\mathbf{Q}^{\pi^{*}}$ such that $\widetilde{X}^{k} \rightarrow \widetilde{X}, \widetilde{Y}^{k} \rightarrow \widetilde{Y}$ a.s. in the uniform norm.

By Assumption 2.3, $\widetilde{Y}^{k}$ and $\widetilde{Y}$ have the same law and $\widetilde{Y}^{k} \rightarrow \widetilde{Y}$ in probability (even a.s.). By Theorem 1 in [1] $F\left(\widetilde{Y}^{k}\right) \rightarrow F(\widetilde{Y})$ in probability.

By continuity of $u_{ \pm}$and the projection $p_{T}(f):=f(T), f \in C([0, T] ; \mathbf{R})$ we also have $u_{ \pm}\left(\left(\widetilde{X}_{T}^{k}-F\left(\widetilde{Y}^{k}\right)\right)_{ \pm}\right) \rightarrow u_{ \pm}\left(\left(\widetilde{X}_{T}-F(\widetilde{Y})\right)_{ \pm}\right)$in probability.

It follows that denoting by $D$ the set of discontinuity points of the cumulative distribution functions of $u_{ \pm}\left(\left(\widetilde{X}_{T}-F(\widetilde{Y})\right)_{ \pm}\right)$, for any $y \in \mathbf{R} \backslash D$ we have

$$
\mathbf{Q}^{\pi^{k}}\left(u_{ \pm}\left(\left(X_{T}^{\pi^{k}}-G^{\pi^{k}}\right)_{ \pm}\right)>y\right) \rightarrow \mathbf{Q}^{\pi^{\star}}\left(u_{ \pm}\left(\left(X_{T}^{\pi^{\star}}-G^{\pi^{\star}}\right)_{ \pm}\right)>y\right)
$$

as $k \rightarrow \infty$.

Since $w_{ \pm}$are continuous, also

$$
w_{ \pm}\left(\mathbf{Q}^{\pi^{k}}\left(u_{ \pm}\left(\left(X_{T}^{\pi^{k}}-G^{\pi^{k}}\right)_{ \pm}\right)>y\right)\right) \rightarrow w_{ \pm}\left(\mathbf{Q}^{\pi^{\star}}\left(u_{ \pm}\left(\left(X_{T}^{\pi^{\star}}-G^{\pi^{\star}}\right)_{ \pm}\right)>y\right)\right)
$$

for $y \notin D$. By Fatou's lemma,

$$
\begin{aligned}
\int_{0}^{\infty} & w_{-}\left(\mathbf{Q}^{\pi^{\star}}\left(u_{-}\left(\left(X_{T}^{\pi^{\star}}-G^{\pi^{\star}}\right)_{-}\right)>y\right)\right) d y \\
& \leqq \liminf _{k} \int_{0}^{\infty} w_{-}\left(\mathbf{Q}^{\pi^{k}}\left(u_{-}\left(\left(X_{T}^{\pi^{k}}-G^{\pi^{k}}\right)_{-}\right)>y\right)\right) d y
\end{aligned}
$$

and, by (2.29) and Fatou's lemma,

$$
\begin{aligned}
\int_{0}^{\infty} & w_{+}\left(\mathbf{Q}^{\pi^{\star}}\left(u_{+}\left(\left(X_{T}^{\pi^{\star}}-G^{\pi^{\star}}\right)_{+}\right)>y\right)\right) d y \\
& \geqq \limsup _{k} \int_{0}^{\infty} w_{+}\left(\mathbf{Q}^{\pi^{k}}\left(u_{+}\left(\left(X_{T}^{\pi^{k}}-G^{\pi^{k}}\right)_{+}\right)>y\right)\right) d y
\end{aligned}
$$

It follows that $V\left(\pi^{\star}\right)=\sup _{\pi \in \bar{\Pi}^{\prime}} V(\pi)$. It is also clear that $\pi^{\star} \in \bar{\Pi}^{\prime}$. Let $\left(a_{t}, b_{t}\right)$ be the A-valued processes associated to $\pi^{\star} \in \bar{\Pi}$ as in Definition 2.4. By Lemma 2.2 there is

$$
\pi^{\prime}=\left(\Omega^{\pi^{\star}}, \mathcal{F}^{\pi^{\star}},\left(\mathcal{F}_{t}^{\pi^{\star}}\right)_{0 \leqq t \leqq T}, \mathbf{P}^{\pi^{\star}}, X_{t}^{\pi^{\prime}}, Y_{t}^{\pi^{\star}},\left(B_{t}^{\pi^{\star}}, W_{t}^{\pi^{\star}}\right)\right)
$$

which is a portfolio value process in the sense of Definition 2.5 and for which

$$
u_{+}\left(\left(X_{T}^{\pi^{\prime}}-G^{\pi^{\prime}}\right)_{+}\right) \geqq u_{+}\left(\left(X_{T}^{\pi^{\star}}-G^{\pi^{\star}}\right)_{+}\right)
$$

Copyright (c) by SIAM. Unauthorized reproduction of this article is prohibited. 
notice that $G^{\pi^{\prime}}=G^{\pi^{*}}$ and $u_{-}\left(\left(X_{T}^{\pi^{*}}-G^{\pi^{*}}\right)_{-}\right) \geqq u_{-}\left(\left(X_{T}^{\pi^{\prime}}-G^{\pi^{\prime}}\right)_{-}\right)$also. Hence $V\left(\pi^{\prime}\right) \geqq \sup _{\pi \in \bar{\Pi}^{\prime}} V(\pi)$. Thus, recalling Remark 2.5, the investment strategy

$$
\widehat{\pi}=\left(\Omega^{\pi^{\star}}, \mathcal{F}^{\pi^{\star}}, \mathbf{P}^{\pi^{\star}},\left\{\mathcal{F}_{t}^{\pi^{\star}}\right\}_{0 \leqq t \leqq T}, X_{t}^{\pi^{\prime}}, Y_{t}^{\pi^{\star}}, \sqrt{m_{t}^{\pi^{\prime}}},\left(B_{t}^{\pi^{\star}}, W_{t}^{\pi^{\star}}\right)\right)
$$

is optimal, i.e., $\sup _{\pi \in \Pi^{\prime}} V(\pi) \leqq V\left(\pi^{\star}\right) \leqq V\left(\pi^{\prime}\right)=V(\widehat{\pi})$ and, obviously, $\widehat{\pi} \in \Pi^{\prime}$. Theorem 2.1 is proved.

3. Extensions. Based on economic considerations, we extend the model that was developed in the last section by allowing the portfolio value process to influence the factor modeled by $Y_{t}$, the influence being "additive." This may be an appropriate model for, e.g., a large investor. Furthermore, a riskless asset with deterministic interest rate $r_{t}$ at time $t$ is included. For the sake of simplicity we will assume that the factor process $Y$ is one-dimensional; the results can be extended to the multidimensional case in a trivial way.

DEFINITION 3.1. $\nu_{t}$ is an $\mathbf{R}$-valued progressively measurable process on the filtered space

$$
\left(C\left([0, T] ; \mathbf{R}^{d}\right), \mathcal{N}_{T},\left\{\mathcal{N}_{t}\right\}_{0 \leqq t \leqq T}\right) .
$$

Similarly, we define the $\mathbf{R}$-valued coefficients $\theta, \lambda, \rho, \kappa$ to have the same measurability.

In this case, the stochastic differential equations of the optimal investment model are given by

$$
\begin{aligned}
d Y_{t} & =\nu(t, Y .) d t+\kappa(t, Y .) d B_{t}+\rho(t, X .) d X_{t} \\
d X_{t} & =\phi_{t} \theta(t, Y .) X_{t} d t+\phi_{t} \lambda(t, Y .) X_{t} d W_{t}+\left(1-\phi_{t}\right) r_{t} X_{t} d t
\end{aligned}
$$

where $\phi_{t} \in[0,1]$ represents the proportion of wealth invested in the stock, $Y$ is an economic factor, and $X$ is the value process of the given portfolio strategy $\phi$. The set $\Pi$ can be defined analogously to Definition 2.2 .

Assumption 3.1. For all $t \geqslant 0$ the growth rate of the stock is greater than the bond's yield, i.e., for all t, $y$.,

$$
\theta(t, y .) \geqq r_{t} \geqq 0 \quad \mathbf{P}^{\pi}-a . s .
$$

The functionals $\theta, \lambda, \rho$ are bounded and path-continuous in the sense of Assumption 2.2. The functionals $\nu$ and $\kappa$ can satisfy a linear growth condition, as in Assumption 2.2 .

Assumption 3.2. The reference point $G$ is a constant.

As in subsection 2.3, we consider a relaxed setting. With this purpose in mind, we define $\theta^{r}(t, y$. $)=\theta(t, y$. $)-r_{t}$. In what follows, $E$ is a $(2 \times 2)$-matrix such that $E^{11}=1$ and $E^{i j}=0$ otherwise.

DeFinition 3.2. We define the following family of sets:

$$
\begin{aligned}
A_{t}(x ., y .)=\{( & (a, b) \in \mathbf{A} \mid a=\frac{1}{2} \kappa^{2}(t, y .) E \\
& +\frac{1}{2} m \lambda^{2}(t, y .) x_{t}^{2}\left(\begin{array}{cc}
\rho^{2}(t, x .) & \rho(t, x .) \\
\rho(t, x .) & 1
\end{array}\right), b=\left(\begin{array}{c}
\nu(t, y .) \\
0
\end{array}\right) \\
& \left.+\left(l x_{t} \theta^{r}(t, y .)+r_{t} x_{t}\right)\left(\begin{array}{c}
\rho(t, x .) \\
1
\end{array}\right), 0 \leqq m \leqq 1,0 \leqq l \leqq \sqrt{m}\right\} .
\end{aligned}
$$

Copyright $@$ by SIAM. Unauthorized reproduction of this article is prohibited. 
The following lemma is crucial: it enables us to use results of [12].

Lemma 3.1. The set $A_{t}(x ., y$.$) is closed, convex, and bounded for each (x ., y.) \in$ $C\left([0, T] ; \mathbf{R}^{2}\right)$ and each $t \geqq 0$.

Proof. Only convexity needs to be checked. Let $0 \leqq \mu \leqq 1$ and $(a, b),(\alpha, \beta) \in$ $A_{t}(x ., y$. $)$; then the convex linear combination $\mu a+(1-\mu) \alpha$ is equal to

$$
\frac{1}{2} \kappa^{2}(t, y .) E+\frac{1}{2}\left(\mu m+(1-\mu) m^{\prime}\right) \lambda^{2}(t, y .) x_{t}^{2}\left(\begin{array}{cc}
\rho^{2}(t, x .) & \rho(t, x .) \\
\rho(t, x .) & 1
\end{array}\right),
$$

and $\mu b+(1-\mu) \beta$ equals

$$
\left(\begin{array}{c}
\nu(t, y .) \\
0
\end{array}\right)+\left(\left(\lambda l+(1-\lambda) l^{\prime}\right) x_{t} \theta^{r}(t, y .)+r_{t} x_{t}\right)\left(\begin{array}{c}
\rho(t, x .) \\
1
\end{array}\right) .
$$

As $\mu l+(1-\mu) l^{\prime} \leqq \mu \sqrt{m}+(1-\mu) \sqrt{m^{\prime}} \leqq \sqrt{\mu m+(1-\mu) m^{\prime}}$, we have $\mu(a, b)+(1-$ $\mu)(\alpha, \beta) \in A_{t}(x ., y$.$) .$

The estimates of Lemma 2.1 can be applied to this case as well; for some $K>0$,

$$
\left\|A_{t}(x, y .)\right\| \leqq K\left(1+\left|x_{t}\right|^{2}\right)
$$

This allows us to apply the results of [12] just as above, using the class of relaxed controls defined in what follows.

Definition 3.3. We say that $\bar{\pi} \in \bar{\Pi}$ if

$$
\bar{\pi}:=\left(\Omega, \mathcal{F},\left\{\mathcal{F}_{t}\right\}_{0 \leqq t \leqq T}, \mathbf{P}, X_{t}, Y_{t},\left(B_{t}, W_{t}\right)\right)
$$

where

(a) $\left(\Omega, \mathcal{F},\left\{\mathcal{F}_{t}\right\}_{0 \leqq t \leqq T}, \mathbf{P}\right)$ is a complete filtered probability space whose filtration satisfies the usual conditions;

(b) the two-dimensional process $\xi_{t}:=\left(B_{t}, W_{t}\right)$ is a two-dimensional standard $\mathcal{F}_{t}$-Wiener process $\left(W_{t}\right.$ is unidimensional $)$

(c) there exists an $\mathbf{A}$-valued, $\mathcal{F} \otimes \mathcal{B}([0, T])$ measurable and $\mathcal{F}_{t}$-adapted process denoted by $\left(a_{t}, b_{t}\right)$ such that

$$
\left(\begin{array}{c}
Y_{t} \\
X_{t}
\end{array}\right)=\left(\begin{array}{c}
y \\
x
\end{array}\right)+\int_{0}^{t} \sqrt{2 a_{s}} d \xi_{s}+\int_{0}^{t} b_{s} d s
$$

(d) for almost all $(\omega, t) \in \Omega \times[0, T]$, we have $\left(a_{t}, b_{t}\right) \in A_{t}(X$.,Y.) (i.e., we can choose a pair $\left(m_{t}, l_{t}\right)$ in a "measurable way").

The vectorial form of (2.4) and (2.5) can be rewritten. Define

$$
\sigma_{t}:=\left(\begin{array}{cc}
\kappa(t, y .) & \rho(t, x .) \sqrt{m_{t}} x_{t} \lambda(t, y .) \\
0 & \sqrt{m_{t}} \lambda(t, y .) x_{t}
\end{array}\right),
$$

and we have that the drift is given by

$$
b_{t}=\left(\begin{array}{c}
\nu(t, y .) \\
0
\end{array}\right)+\left(x_{t} l_{t} \theta^{r}(t, y .)+x_{t} r_{t}\right)\left(\begin{array}{c}
\rho(t, x .) \\
1
\end{array}\right) .
$$

Given a relaxed control $\bar{\pi}, X_{t}, Y_{t}$ are $\mathcal{F} \otimes \mathcal{B}([0, T])$-measurable and $\mathcal{F}_{t}$-adapted such that for all $t \in[0, T]$

$$
\begin{aligned}
d Y_{t} & =\nu(t, Y .) d t+\kappa(t, Y .) d B_{t}+\rho(t, X .) d X_{t} \\
d X_{t} & =\left[l_{t}\left(\theta(t, Y .)-r_{t}\right) X_{t}+r_{t} X_{t}\right] d t+\sqrt{m_{t}} \lambda(t, Y .) X_{t} d W_{t}
\end{aligned}
$$

Copyright $\odot$ by SIAM. Unauthorized reproduction of this article is prohibited. 
The proof of the following result follows closely that of Theorem 2.1.

TheOREm 3.1. Let Assumptions 2.3, 2.4, 3.1, and 3.2 hold. The problem (2.9) is well-posed, and $\Pi^{\prime} \neq \varnothing$ (the identically zero strategy belongs to $\Pi^{\prime}$, where $\Pi^{\prime}$ is defined analogously to subsection 2.2). There is $\widehat{\pi} \in \Pi^{\prime}$ such that the supremum in (2.9) is attained.

4. Appendix. Some proofs of auxiliary results are included in this appendix.

Proof of Proposition 2.1. Fix $\bar{\pi} \in \bar{\Pi}$, and let $\zeta_{t}=\left(X_{t}, Y_{t}\right)$ and $\xi_{t}=\left(B_{t}, W_{t}\right)$ be their corresponding processes. Suppose $m \geqq 2$. The notation $|\cdot|$ will be used to denote a Euclidean norm in spaces of various dimensions. Moreover, by localization, we may suppose that the processes are bounded. Then

$$
\left|\zeta_{t}\right|^{m}=\left[\left(X_{t}\right)^{2}+\left|Y_{t}\right|^{2}\right]^{m / 2} \leqq 2^{m / 2-1}\left[\left|X_{t}\right|^{m}+\left|Y_{t}\right|^{m}\right],
$$

so we prove the result by estimating $\sup _{t \leqq T}\left|Y_{t}\right|^{m}$ and $\sup _{t \leqq T}\left|X_{t}\right|^{m}$ separately. Set $b_{s}^{(2)}=l_{t} \theta_{t}(Y.) X_{t}, b_{s}^{(1)}=\nu_{t}(Y$.$) , and \sigma_{s}^{(1)}=\left(\mathbf{0}^{\prime}, m_{t} \lambda_{t}(Y.) X_{t}\right), \sigma_{s}^{(2)}=\left(\kappa_{t}(Y),. \mathbf{0}\right)$; here $\mathbf{0} \in \mathbf{R}^{d \times 1}$. Then

$$
\mathbf{E}_{\pi}\left[\sup _{t \leqq T}\left|X_{t}\right|^{m}\right] \leqq 3^{m-1}\left(|x|^{m}+\mathbf{E}_{\pi}\left(\int_{0}^{T}\left|b_{s}^{(2)}\right| d s\right)^{m}+\mathbf{E}_{\pi}\left[\sup _{t \leqq T}\left|\int_{0}^{t} \sigma_{s}^{(2)} d \xi_{s}\right|^{m}\right]\right) .
$$

By the Burkholder-Davis-Gundy inequality

$$
\begin{aligned}
\mathbf{E}_{\pi}\left[\sup _{t \leqq T}\left|X_{t}\right|^{m}\right] \leqq 3^{m-1} & \left(|x|^{m}+\mathbf{E}_{\pi}\left(\int_{0}^{T}\left|b_{s}^{(2)}\right| d s\right)^{m}\right. \\
& \left.+C_{m} \mathbf{E}_{\pi}\left[\left.\left.\left|\int_{0}^{T}\right| \sigma_{s}^{(2)}\right|^{2} d s\right|^{m / 2}\right]\right)
\end{aligned}
$$

for some $C_{m}>0$. We can apply Hölder's inequality to the first integral

$$
\begin{gathered}
\left(\int_{0}^{T}\left|b_{s}^{(2)}\right| d s\right)^{m} \leqq T^{m-1} \int_{0}^{T}\left|b_{s}^{(2)}\right|^{m} d s \\
\left.\left.\left|\int_{0}^{T}\right| \sigma_{s}^{(2)}\right|^{2} d s\right|^{m / 2} \leqq T^{m / 2-1} \int_{0}^{T}\left|\sigma_{s}^{(2)}\right|^{m} d s ;
\end{gathered}
$$

here $\left|\sigma_{s}^{(2)}\right|^{m}=m_{t}^{m}\left|\lambda_{t}(Y .)\right|^{m} X_{t}^{m}$ and $\left|b_{s}^{(2)}\right|^{m}=l_{t}^{m} \theta_{t}^{m}(Y.) X_{t}^{m}$,

$$
\int_{0}^{T}\left|\sigma_{s}^{(2)}\right|^{m} d s \leqq \int_{0}^{T}\left(\left|\lambda_{s}(Y .)\right|^{2} X_{s}^{2} m_{s}^{2}+\left\|\kappa_{s}(Y .)\right\|^{2}\right)^{m / 2} d s .
$$

By (2.20),

$$
\int_{0}^{T}\left|\sigma_{s}^{(2)}\right|^{m} d s \leqq 2^{m-1} K^{m} \mathbf{E}_{\pi}\left[\int_{0}^{T}\left(1+\sup _{r \leqq s}\left|\zeta_{r}\right|\right)^{m} d s\right]
$$

and finally we obtain the estimate

$$
\mathbf{E}_{\pi}\left[\sup _{t \leqq T}\left|X_{t}\right|^{m}\right] \leqq 3^{m-1}\left(|x|^{m}+g_{1}(T)+g_{2}(T) \mathbf{E}_{\pi}\left[\int_{0}^{T} \sup _{t \leqq s}\left|\zeta_{t}\right|^{m} d s\right]\right),
$$

Copyright (c) by SIAM. Unauthorized reproduction of this article is prohibited. 
with $g_{1}(T)=C_{m}^{\prime} T^{m / 2}$ and $g_{2}(T)=K^{\prime}\left(T^{m / 2-1}+T^{m-1}\right)$. Similarly,

$$
\mathbf{E}_{\pi}\left[\sup _{t \leqq T}\left|Y_{t}\right|^{m}\right] \leqq 3^{m-1}\left(|y|^{m}+h_{1}(T)+h_{2}(T) \mathbf{E}_{\pi}\left[\int_{0}^{T} \sup _{t \leqq s}\left|\zeta_{t}\right|^{m} d s\right]\right)
$$

and $h_{1}(T)=C_{m}^{\prime \prime}\left(T^{m}+T^{m / 2}\right)$ and $h_{2}(T)=K^{\prime \prime}\left(T^{m / 2-1}+T^{m-1}\right)$.

Denoting $f(T)=\mathbf{E}\left[\sup _{t \leqq T}\left|\zeta_{t}\right|^{m}\right]$ and adding the inequalities in (4.3) and (4.2), we see that the following inequality holds:

$$
f(T) \leqq G_{1}(T)+G_{2}(T) \int_{0}^{T} f(s) d s
$$

with suitable functions $G_{1}, G_{2}$. By considering the derivative of $\exp \left(-\int_{0}^{T} G_{2}(s) d s\right) \times$ $\int_{0}^{T} f(s) d s$ and the condition (4.4), one can see that

$$
\begin{aligned}
\frac{d}{d T}(\exp & \left.\left(-\int_{0}^{T} G_{2}(s) d s\right) \int_{0}^{T} f(s) d s\right) \\
\quad & \exp \left(-\int_{0}^{T} G_{2}(s) d s\right)\left(f(T)-G_{2}(T) \int_{0}^{T} f(s) d s\right) \\
\quad & \leqq \exp \left(-\int_{0}^{T} G_{2}(s) d s\right) G_{1}(T),
\end{aligned}
$$

and integrating the inequality,

$$
\int_{0}^{T} f(s) d s \leqq \int_{0}^{T} \exp \left(\int_{s}^{T} G_{2}(r) d r\right) G_{1}(s) d s
$$

thus, (4.4) implies that

$$
f(T) \leqq G_{1}(T)+G_{2}(T) \int_{0}^{T} \exp \left(\int_{s}^{T} G_{2}(r) d r\right) G_{1}(s) d s<\infty .
$$

The general case follows from localization and an application of the monotone convergence theorem. The case $0<m<2$ follows from the monotonicity of the norms. Proposition 2.1 is proved.

Proof of Proposition 2.2. As in Proposition 2.1, it is enough to show a similar estimate (2.24) for each of the coordinates $X_{t}$ and $Y_{t}$. That means

$$
\mathbf{E}_{\bar{\pi}}\left|Y_{t}-Y_{s}\right|^{\eta} \leqq K_{1}|t-s|^{\eta / 2} \quad \text { and } \quad \mathbf{E}_{\bar{\pi}}\left|X_{t}-X_{s}\right|^{\eta} \leqq K_{2}|t-s|^{\eta / 2} .
$$

By Assumption 2.2 the following inequalities are a simple consequence of the Burkholder-Davis-Gundy inequality and well-known moments estimates:

$$
\begin{aligned}
\mathbf{E}_{\bar{\pi}}\left|Y_{t}-Y_{s}\right|^{\eta} \leqq & 2^{\eta-1}\left(\mathbf{E}_{\bar{\pi}}\left(\int_{s}^{t}\left|\nu_{r}(Y .)\right| d r\right)^{\eta}\right. \\
& \left.+\mathbf{E}_{\bar{\pi}}\left(\sup _{s \leqq u \leqq t}\left|\int_{s}^{u} \kappa_{r}(Y .) d B_{r}\right|^{\eta}\right)\right), \\
\leqq & 2^{\eta-1}\left(M^{\eta}|t-s|^{\eta}+C_{\eta} \mathbf{E}_{\bar{\pi}}\left(\int_{s}^{t}\left\|\kappa_{r}(Y .)\right\|^{2} d r\right)^{\eta / 2}\right) .
\end{aligned}
$$

Copyright (c) by SIAM. Unauthorized reproduction of this article is prohibited. 
Thus

$$
\mathbf{E}_{\bar{\pi}}\left|Y_{t}-Y_{s}\right|^{\eta} \leqq 2^{\eta-1}\left(M^{\eta} T^{\eta / 2}+C_{\eta} M^{\eta}\right)|t-s|^{\eta / 2} .
$$

The following second estimate relies upon Proposition 2.1:

$$
\begin{aligned}
\mathbf{E}_{\bar{\pi}}\left|X_{t}-X_{s}\right|^{\eta} \leqq & 2^{\eta-1}\left(\mathbf{E}_{\bar{\pi}}\left(\int_{s}^{t}\left|l_{r} \theta_{r}(Y .) X_{r}\right| d r\right)^{\eta}\right. \\
& \left.+\mathbf{E}_{\bar{\pi}}\left(\sup _{s \leqq u \leqq t}\left|\int_{s}^{r} \lambda_{r}(Y .) \sqrt{m_{r}} X_{r} d W_{r}\right|^{\eta}\right)\right) \\
\leqq & 2^{\eta-1} M^{\eta}\left(\mathbf{E}_{\bar{\pi}}\left(\int_{s}^{t}\left|X_{r}\right| d r\right)^{\eta}+C_{\eta} \mathbf{E}_{\bar{\pi}}\left(\left|\int_{s}^{t} X_{r}^{2} d r\right|^{\eta / 2}\right)\right) .
\end{aligned}
$$

So

$$
\mathbf{E}_{\bar{\pi}}\left|X_{t}-X_{s}\right|^{\eta} \leqq 2^{\eta-1} M^{\eta}\left((t-s)^{\eta} \mathcal{N}(\eta, T)+(t-s)^{\eta / 2} \mathcal{N}(\eta, T)\right)=K_{3}|t-s|^{\eta / 2},
$$

where

$$
K_{3}=2^{\eta-1} M^{\eta} \mathcal{N}(\eta, T)\left(T^{\eta / 2}+1\right) \quad \text { and } \quad \mathcal{N}(\eta, T)
$$

is the upper bound of $\sup _{\bar{\pi} \in \bar{\Pi}} \mathbf{E}_{\bar{\pi}}\left[\sup _{t \leqq T}\left|X_{t}\right|^{\eta}\right]$ in Proposition 2.1. Note that the constants do not depend on $\bar{\pi}$, as neither $M$ nor $\mathcal{N}(\eta, T)$ do. Proposition 2.2 is proved.

\section{REFERENCES}

[1] M. Barlow, M. Émery, F. Knight, S. Song, And M. Yor, Autour d'un théoreme de Tsirelson sur des filtrations browniennes et non browniennes, in Séminaire de Probabilités XXXII, Lecture Notes in Math. 1686, Springer, Berlin, 1998, pp. 264-305.

[2] D. Bernoulli, Theoriae Novae de Mensura Sortis, Commentarii Academiae Scientiarum Imperialis Petropolitanae, Vol. V, 1738. Translated by L. Sommer as "Exposition of a New Theory on the Measurement of Risk," Econometrica, 22 (1954), pp. 23-36.

[3] A. B. Berkelaar, R. Kouwenberg, and T. Post, Optimal portfolio choice under loss aversion, Rev. Econom. Statist., 86 (2004), pp. 973-987.

[4] F. BlaCK, Studies of stock market volatility changes, in Proceedings of the American Statistical Association, Business and Economic Statistics Section, American Statistical Association Washington, DC, 1976, pp. 177-181.

[5] L. Campi AND M. Del Vigna, Weak insider trading and behavioral finance, SIAM J. Financial Math., 3 (2012), pp. 242-279.

[6] L. Carassus AND M. RÁsonyi, On optimal investment for a behavioral investor in multiperiod incomplete markets, Math. Finance, 25 (2015), pp. 115-153.

[7] G. Carlier and R.-A. Dana, Optimal demand for contingent claims when agents have law invariant utilities, Math. Finance, 21 (2011), pp. 169-201.

[8] A. Cherny and D. P. Madan, New Measures for Performance Evaluation, Rev. Financial Stud., 22 (2009), pp. 2571-2606.

[9] J.-P. Fouque, G. Papanicolau, and R. Sircar, Derivatives in Financial Markets with Stochastic Volatility, Cambridge University Press, Cambridge, UK, 2000.

[10] H. Jin AND X. Y. Zhou, Behavioural portfolio selection in continuous time, Math. Finance, 18 (2008), pp. 385-426.

[11] D. Kahneman and A. Tversky, Prospect theory: An analysis of decision under risk, Econometrica, 47 (1979), pp. 263-291.

[12] N. V. KRYLOV, A supermartingale characterization of sets of stochastic integrals and applications, Probab. Theory Relat. Fields, 123 (2002), pp. 521-552.

[13] N. V. KRylov AND R. Liptser, On diffusion approximation with discontinuous coefficients, Stochastic Proc. Appl., 102 (2002), pp. 235-264.

Copyright (c) by SIAM. Unauthorized reproduction of this article is prohibited. 
[14] J. Quiggin, A Theory of Anticipated Utility, J. Economic and Behavioral Organization, 3 (1982), pp. 323-343.

[15] M. RÁsonyi And A. M. Rodrigues, Optimal portfolio choice for a behavioural investor in continuous-time markets, Ann. Finance, 9 (2013), pp. 291-318.

[16] C. Reichlin, Utility maximization with a given pricing measure when the utility is not necessarily concave, Math. Financial Economics, 7 (2013), pp. 531-556.

[17] C. Reichlin, Non-concave Utility Maximization: Optimal Investment, Stability and Applications, Ph.D. thesis, Diss. ETH 20749, ETH Zürich, 2012.

[18] A. Tversky and D. Kahneman, Advances in prospect theory: Cumulative representation of uncertainty, J. Risk Uncertainty, 5 (1992), pp. 297-323.

[19] J. von Neumann and O. Morgenstern, Theory of Games and Economic Behavior, Princeton University Press, Princeton, NJ, 1944.

Copyright (c) by SIAM. Unauthorized reproduction of this article is prohibited. 